\title{
Session 3: Therapeutic Applications II
}

\author{
Wednesday 14th April 2010. Moderator: Zdenka L. Jonak
}

\section{[14.00-14.30]}

'Development of therapeutic antibodies: Challenges and opportunities'

Yan $\mathrm{Wu}$

NovartisBiologics, Shanghai, China

To date, the most difficult challenge to antibodybased therapy for a variety of diseases, particularly for cancer, is the balance of robust efficacy and minimal side effects on normal tissues. In the case of cancer intervention, it is highly desirable to have a therapeutics controlling metastasis that accounts for most death of cancer patients and enhancing antitumor immunity that is seriously weakened in patients with cancer. Antibody-directed therapeutics has been primarily focused on targeting a specific molecule that is functional in the pathogenesis of diseases. Antibody combination and bispecific antibody targeting disorder-associated cells or factors have been explored in the past years. Alternatively, the development of a therapeutic antibody targeting multiple molecules that involve in the pathogenesis and progression of one or more disease types will be also preferable.

\section{[14.30-14.50]}

'Clinical experience in a phase IIa trail with the human monoclonal IgM antibody Panobacumab in ventilator associated pneumonia caused by P.aeruginosa'

Michael Rudolf

Kenta Biotech AG, Bern, Switzerland

Kenta Biotech, founded in 2006 in Switzerland, is focusing on the discovery and development of fully human monoclonal antibodies for the treatment of intensive care patients with life-threatening infectious diseases. Kenta Biotech employs its proprietary $\mathrm{MAbIg} \mathrm{X}^{\circledR}$ technology platform, which enables the generation of human hybridoma cells from con- valescent patients, for the selection of antibodies of all isotypes specific for bacterial targets. Moreover MAbIgX ${ }^{\circledR}$ derived human hybridoma clones reliably and persistantly grow in synthetic media without the additions of animal derived components. Kenta has developed a portfolio of several antibodies targeting $P$. aeruginosa, S. aureus and A. baumannii for the treatment of severe nosocomial infections.

The lead product, Panobacumab, a fully human IgM which efficiently opsonizes $P$. aeruginosa IATS serotype $\mathrm{O} 11$ bacterial cells and mediates complementdependent phagocytosis in vitro, has recently completed a clinical phase IIA trial in ventilator associated pneumonia patients. The aim of the international multicenter study was to evaluate safety, tolerability, pharmacokinetics, clinical and microbiological outcome. Panobacumab was safe and well tolerated in high risk patients. The PK of the human IgM in patients was comparable to PK data generated in healthy subjects. Most important, none of the evaluable patients completing the full treatment cycle died during the 30 day observation period, although based on APACHE II scores a mortality of $25 \%$ was predicted. Adverse events were mostly self-limited and unrelated to the study drug. Finally, the application of the IgM mAb had no detrimental effect on complement or any laboratory data. The presence of Panobacumab in BAL fluids demonstrated the potential of the IgM to reach the alveolar space in the inflamed lung and therefore to efficiently target $P$. aeruginosa causing VAP.

For future treatment Kenta Biotech is developing additional monoclonal IgM antibodies targeting individual serotypes of $P$. aeruginosa to broaden the coverage to all $P$. aeruginosa induced VAP in ICU patients. 
[14.50-15.10]

'Immunological approaches to preventing and treating Alzheimer's disease'

Andrew Hiatt ${ }^{\mathrm{a}}$ and Alena Savonenko ${ }^{\mathrm{b}}$

a Mapp Biopharmaceutical Inc., San Diego, CA, USA

${ }^{\mathrm{b}}$ Johns Hopkins University School of Medicine, Baltimore, MA, USA

The progression from pre- to post-symptomatic Alzheimer's disease (AD) involves the deposition of the $\mathrm{A} \beta$ brain amyloid peptide (as well as many other events), followed by a transition to a pro-inflammatory, neurotoxic brain environment. We have used transgenic APP/PS1 mice that express human $\mathrm{A} \beta$ to explore different types of immunological intervention. These mice develop measurable cognitive deficit at 6 months of age. First, we isolated a group of mAbs against internal epitopes of $\mathrm{A} \beta$. These mAbs were selected to have high affinity for the soluble, oligomeric forms of $\mathrm{A} \beta$ and were tested in 9-10 month old mice given a series of cognitive tasks. By and large, IP injection of these mAbs over 6 weeks resulted in a partial but significant amelioration of spatial reference, episodic-like, and working memory deficits. Our second approach involved injection of high doses of total murine $\operatorname{IgG}(\mathrm{mIgG})$, also in 9-10 month old mice. Complete amelioration was observed in long-term reference memory however partial amelioration was observed in the other cognitive tests. A protocol of weekly injections from 2-6 months of age resulted in complete amelioration in all cognitive tests. Equivalent experiments with mAbs are in progress. The results suggest that the efficacy of $\mathrm{mIgG}$ depends largely on the timing and duration of injection similar to the efficacy of $\mathrm{mCSF}$ in the same mice. Similar observations have been made regarding NSAIDs in humans. Moreover, the mechanism of action of $\mathrm{mIgG}$ may involve interaction with splenic regulatory macrophages capable of eliciting anti-inflammatory cellular events at peripheral sites of inflammation. Since $\mathrm{mIgG}$ also contains detectible anti-A $\beta$ a mechanism of specific binding cannot be ruled out.

[15.10-15.30]

'Pre-clinical development of a novel therapeutic antibody to treat pancreas and colorectal cancers' Andrew Bristol, Philip Arlen, Judith Kantor, Janos Luka, Olga Saric, Rishab Gupta, Myron Arlen Neogenix Oncology Inc., Rockville, MA, USA
NPC-1C is a chimeric monoclonal antibody being developed as a novel biological treatment for pancreatic and colorectal cancers. This antibody was selected from a panel of hybridomas generated from mice immunized with semi-purified membrane-associated proteins derived from biologically screened, pooled human allogeneic colon cancer tissues. NPC-1C appears to recognize an aberrantly glycosylated form of MUC5AC expressed specifically by human pancreatic and colorectal tumor tissues and cell lines. NPC-1C does not cross-react with other tumor cell lines or normal human tissues, however, except for occasional, weak binding to certain GI tract tissues, which may indicate a premalignant state.

In vitro, the NPC-1C antibody exhibits specific $\mathrm{AD}$ $\mathrm{CC}$ activity against human colon and pancreatic tumor cells, but not against control tumor cell lines. In vi$v o$, the anti-tumor efficacy of NPC-1C was tested using pre-established subcutaneous human tumor xenograft models. The data showed significant, and reproducible, anti-tumor action, including complete tumor regressions. The clinical application for this antibody was bolstered by approximately $45 \%$ of human pancreatic and colon tumor tissues stained positively with NPC$1 \mathrm{C}$.

The pre-clinical toxicity profile of NPC-1C in normal BALB/c mice demonstrated little, if any, toxicity following single or multiple intravenous injections of $10 \mathrm{mg} / \mathrm{kg}, 50 \mathrm{mg} / \mathrm{kg}$, or $100 \mathrm{mg} / \mathrm{kg}$ of clinicalgrade NPC-1C antibody. The bio-distribution of radiolabeled NPC-1C in mice with pre-established subcutaneous human tumors revealed specific accumulation of NPC-1C at the tumor site, with little or no binding or accumulation in several major organ systems. Finally, the cytokine and antibody responses to NPC-1C were studied in the pre-clinical mouse model. Although antiNPC-1C antibodies were detected (as expected) in a time-dependent fashion, there were no changes detect in Type I (IL-2 or g-IFN) and Type II (IL-4 or IL-5) cytokines in response to up to $100 \mathrm{mg} / \mathrm{kg}$ of NPC-1C either 3 days or 14 days after intravenous administration.

The pre-clinical development of NPC-1C indicates that it is safe and efficacious, and suggest that it should be well-tolerated and may have clinical activity in patients whose tumor expresses the altered MUC5AC epitope. The FDA has approved an Investigational New Drug application to initiate a Phase I clinical trial with NPC-1C for patients with advanced pancreatic and colorectal cancer. 
[15.30-15.50]

'Raxibacumab for the treatment of inhalational anthrax'

Larry Lo

Human Genome Sciences Inc., Rockville, MA, USA

Raxibacumab is a fully human monoclonal antibody (IgG1 $\lambda \mathrm{p}$ that Human Genome Sciences (HGS) discovered and developed for the treatment of inhalation anthrax. Raxibacumab is a first-in-class treatment that targets anthrax toxins after they are released by the bacteria into the blood and tissues. In April 2009, HGS completed the delivery of 20,000 doses of raxibacumab to the U.S. Strategic National Stockpile. In July 2009, HGS received a second order for 45.000 doses of raxibacumab from the U.S. Government, to be delivered over a period of three years, beginning near the end of 2009. Raxibacumab is also the first biological drug seeking the approval under Animal Rule. This presentation will cover from initial discovery of antibody, including selection/screening and characterization, in vitro/in vivo preclinical development to animal efficacy study. 